\title{
Molecular characterization of rat cvHsp/HspB7 in vitro and its dynamic molecular architecture
}

\author{
ZEHONG YANG, YAO WANG, YONGZHI LU and XIAOJUN ZHAO \\ Nanomedicine Laboratory, Institute for Nanobiomedical Technology and Membrane Biology, and \\ West China Hospital, Sichuan University, Chengdu 610041, P.R. China
}

Received June 21, 2010; Accepted September 28, 2010

DOI: $10.3892 / \mathrm{mmr} .2010 .382$

\begin{abstract}
Cardiovascular heat shock protein (cvHsp) is an abundant and selectively expressed component in cardiac tissue with putative molecular functionality. The most prominent feature of cvHsp is the characteristic $\alpha$-crystallin domain, which makes it a member of the small heat shock protein (sHsp) family. In the present study, we cloned and expressed the cvHsp gene, purified cvHsp to homogeneity, and characterized its structural and molecular properties. The cvHsp mainly consisted of $\beta$-sheets and randomly coiled secondary structural elements, and in addition possessed variable tertiary structures and several solventexposed hydrophobic patches on its molecular surface. The purified cvHsp existed as a mixture of dimers and oligomers containing 12 monomers, and exhibited subunit exchange capacity when labeled with AIAS and LYI. In total, the cvHsp was characterized as having an oligomeric molecular architecture, and displayed various properties common to the sHsp family. The cvHsp gene may have physiologically important implications in the cardiac stress response.
\end{abstract}

\section{Introduction}

Heat shock proteins (Hsps) are highly conserved and play an important role in the folding, intracellular localization and degradation of proteins. They are generally classified based on their molecular mass, and include Hsp100, Hsp90, Hsp70 and Hsp60, and the small heat shock proteins (sHsps), the monomers of which range from 12 to $43 \mathrm{kDA}$. However, most sHsps form a large oligomeric architecture of 150-800 kDA. The sHsps are the most diverse of the Hsp families, and are ubiquitous molecular chaperones that participate in various physiological processes underlying stress tolerance, longevity

Correspondence to: Dr Xiaojun Zhao, Nanomedicine Laboratory, Institute for Nanobiomedical Technology and Membrane Biology, and West China Hospital, Sichuan University, Science Park No.1, Ke Yuan 4th Street, Gao Peng Road, Hi-tech Industrial Development Zone, Chengdu 610041, P.R. China

E-mail: xiaojunz@mit.edu; xz299@cornell.edu

Key words: cardiovascular heat shock protein, molecular architecture, small heat shock proteins, subunit exchange and aging, and apoptosis in most organisms (1-3). Most of the conservation between sHsps occurs in the region known as the $\alpha$-crystallin domain, a stretch of $80-100$ amino acids that is generally located at the C-terminus, and is the signature of an sHsp. A number of studies have shown that this domain is important for subunit-subunit interactions, which lead to molecular oligomeric architecture $(3,4)$. In general, mammalian sHsps form polydispersed oligomers in vitro and possess a high molecular mass architecture in the range of 4-40 subunits, which displays dynamic variable quaternary structures with subunits that freely and rapidly exchange between the oligomers. This dynamic architecture of sHsps confers a markedly higher capacity compared to other chaperones, which can bind substrates or client proteins with a 1-to-1 stoichiometry $(5,6)$.

Some members of the sHsp family have been well characterized, such as HspB1, $\alpha$ B-crystallin (HspB5) and HspB6, but the structural and functional properties of various sHsps, such as HspB9, HspB10 and cardiovascular heat shock protein (cvHsp) (also known as HspB7) are not completely understood $(3,4,7,8)$. Of the 10 sHsps identified to date, cvHsp was found to be selectively and highly expressed in myogenic tissue. Intriguingly, cvHsp expression, which is particularly high in the heart $(0.3 \%$ of the total RNA), moderate in skeletal muscle, and low in aortas or adipose tissues, is involved in a variety of cardiac pathologies. The cvHsp gene was mapped to 1p36.23-p34.3 between markers D1S434 and D1S507 on chromosome 1. Amino acid residues 56-119 of cvHsp are important for its specific interaction with the C-terminal tail of $\alpha$-filamin and actin-binding protein 280 (9). A novel finding demonstrated that cvHsp constitutively localizes to SC35 splicing speckles, driven by its N-terminal serine-rich region, which displays homology to an SR motif (10). Recently, another investigation identified a genetic association between cvHsp polymorphisms and sporadic systolic heart failure (11). However, the biochemical properties and molecular architecture of cvHsp have yet to be elucidated.

In the present study, we cloned and expressed the rat cvHsp gene, and characterized the secondary, tertiary and quaternary structure of recombinant cvHsp. Additionally, we identified the molecular subunit exchange capacity of cvHsp using the fluorescence resonance energy transfer (FRET) method. The results provided various insights into the molecular functionality of the rat cvHsp gene. 


\section{Materials and methods}

Cloning of the rat cvHsp gene and construction of the pET21a-cvHsp plasmid. Total RNA was isolated from $40 \mathrm{mg}$ of rat ventricle tissue with the RNA Isolation and Purification kit (Watson Biotech Inc., Shanghai, China), and first-strand cDNA was rapidly synthesized by the RevertAid ${ }^{\mathrm{TM}}$ First Strand cDNA Synthesis kit (Fermentas, USA). The cvHsp gene was amplified by polymerase chain reaction (PCR) using a forward primer (5'-GGGAATTCUCATATGUTCCTCCTC TTCTTCCTCCT-3') containing an NdeI restriction site (underlined) and a reverse primer (5'-AAAGAATTCTTA GATTTTTATCTCCGTCCGGAAG-3') containing an EcoRI site (underlined). The DNA fragment corresponding to the cvHsp gene was inserted into the pMD18-T simple vector. The corresponding fragment was ligated into the NdeI and EcoRI restriction sites of the pET21a vector to construct the pET21acvHsp plasmid, which was used to express the full-length cvHsp gene. The coding sequence and the reading frame of the pET21a-cvHsp plasmid were confirmed by DNA sequencing (Invitrogen, Shanghai, China).

cvHsp overexpression and purification. The pET21a-cvHsp plasmid was transformed into the competent Rosetta (DE3) pLysS E. coli strain, which was grown at $37^{\circ} \mathrm{C}$ in LB medium containing ampicillin $(100 \mu \mathrm{g} / \mathrm{ml})$ and chloramphenicol $(34 \mu \mathrm{g} / \mathrm{ml})$ until the $\mathrm{OD}_{600}$ of the culture reached $0.7 \mathrm{AU}$ (absorbance units). The temperature was then changed to $30^{\circ} \mathrm{C}$, and isopropyl $\beta$-D-thiogalactopyranoside (IPTG) was added to $1 \mathrm{mM}$ to induce overexpression of the cvHsp gene. The cultures were incubated for an additional $4 \mathrm{~h}$. Cells were harvested by centrifugation $\left(12,000 \mathrm{x} \mathrm{g}, 20 \mathrm{~min}, 4^{\circ} \mathrm{C}\right)$, and cell pellets were suspended in lysis buffer $[50 \mathrm{mM}$ Tris- $\mathrm{HCl}$, pH 7.4, 1 mM ethylenediaminetetraacetic acid (EDTA), $1 \mathrm{mM}$ phenylmethylsulfonyl fluoride (PMSF)] and lysed by ultrasonication. The supernatants were obtained by centrifugation $\left(50,000 \times \mathrm{g}, 30 \mathrm{~min}, 4^{\circ} \mathrm{C}\right)$ and subjected to fractional precipitation by stepwise addition of $0-100 \%$ saturated ammonium sulfate in $20 \%$ increments (each step was maintained for $30 \mathrm{~min}$ at $4^{\circ} \mathrm{C}$ ). The fraction containing cvHsp was subjected to dialysis against $20 \mathrm{mM}$ Tris- $\mathrm{HCl}$ buffer $(\mathrm{pH}$ 8.0) and applied to a Q-Sepharose anion exchange chromatography column, where the peak containing cvHsp was eluted with a gradient of $0-1.0 \mathrm{M} \mathrm{NaCl}$. Fractions containing cvHsp were pooled and dialyzed overnight in $50 \mathrm{mM}$ phosphate-buffered saline (PBS, pH 7.4) containing $0.15 \mathrm{M} \mathrm{NaCl}$. The pooled fractions were directly applied to a Superdex75 10/300GL column (GE Biosciences). Fractions containing cvHsp were separated by SDS-PAGE and dialyzed in $50 \mathrm{mM}$ PBS (pH 7.4) containing $1 \mathrm{mM}$ DTT and stored at $-80^{\circ} \mathrm{C}$ until use. The concentration of cvHsp was analyzed using an extinction coefficient $\left(\varepsilon_{280 \mathrm{~nm}}\right)$ of 0.725 for $1 \mathrm{mg} / \mathrm{ml}$ protein solution. The extinction coefficient was calculated using the method described by Pace et al (12).

Circular dichroism (CD) spectroscopy. The structure of cvHsp was measured by far- and near-UV CD spectra in a Model $400 \mathrm{CD}$ spectropolarimeter (Aviv Inc., USA). The far-UV CD signal was collected in the range of 195-250 nm, and the near-UV CD signal was collected in the range of $250-320 \mathrm{~nm}$. The protein sample was prepared at a concentration of
$0.20 \mathrm{mg} / \mathrm{ml}$ in $50 \mathrm{mM}$ PBS (pH 7.4). The scan temperatures were set at 20,37 and $50^{\circ} \mathrm{C}$. Data were collected at step sizes of $1 \mathrm{~nm}$ with an average time of $5 \mathrm{sec}$ in a quartz cuvette with an optical path length of $2 \mathrm{~mm}$. The spectra were recorded at a scan speed of $1 \mathrm{~nm} / \mathrm{sec}$. The reported CD spectra are the average of three scans in millidegrees that were converted to molar ellipticity. The secondary structural content was estimated using CDPro deconvolution software (13).

Fluorescence spectroscopy. The intrinsic tyrosine fluorescence of cvHsp was analyzed with a Hitachi F-7000 spectrophotometer (Hitachi Corp. Japan) equipped with a thermostatted cell holder. The protein sample was diluted to $0.45 \mathrm{mg} / \mathrm{ml}$ in $20 \mathrm{mM}$ PBS (pH 7.4) containing $0.15 \mathrm{M} \mathrm{NaCl}$. The excitation wavelength was set at $285 \mathrm{~nm}$, and the emission spectrum was recorded between 310 and $390 \mathrm{~nm}$, with an emission slit of $3 \mathrm{~nm}$. For the 1,1'-bi(4-anilino)naphthalenesulfonic acid (bis-ANS) binding assay, the hydrophobic probe, bis-ANS, was added to a final concentration of $10 \mu \mathrm{M}$ in cvHsp solution of $100 \mu \mathrm{l}$ and incubated for $20 \mathrm{~min}$ at room temperature. The excitation wavelength was set at $385 \mathrm{~nm}$. The emission spectrum was recorded from 400 to $600 \mathrm{~nm}$, with the excitation and emission band slits both set to $3 \mathrm{~nm}$.

Gel filtration chromatography and subunit exchange. The oligomeric size of cvHsp was measured by analytical gel filtration chromatography. A Superdex75 10/300GL prepacked gel filtration column (10x300 mm, 24-ml bed volume; GE Bioscience) was used with an Akta Purifier 10 system (GE Bioscience). The column was previously equilibrated with $50 \mathrm{mM}$ PBS (pH 7.4) containing $0.15 \mathrm{M} \mathrm{NaCl}$, and $200 \mu \mathrm{l}$ of cvHsp $(0.45 \mathrm{mg} / \mathrm{ml})$ was loaded at a flow rate of $0.30 \mathrm{ml} /$ min in equilibration buffer with detection wavelengths of 280 and $215 \mathrm{~nm}$. Molecular mass markers (thyroglobulin, ferritin, human IgG, BSA and lysozyme; all from Sigma) were used to calibrate the column. The column efficiency was measured to be higher than 25,000 plates/meter.

For subunit exchange reactions, cvHsp was diluted to a concentration of $1 \mathrm{mg} / \mathrm{ml}$ in $20 \mathrm{mM}$ PBS (pH 7.8) containing $0.15 \mathrm{M} \mathrm{NaCl}$. Subunit exchange kinetics were measured according to the method described by Bova et al $(14,15)$ and Ahmad et al (16). Briefly, the dipotassium salt of Lucifer yellow iodoacetamide (LYI; Invitrogen, Shanghai, China) and the disodium salt of 4-acetamido-4'-((iodoacetyl)amino) stilbene-2,2'-disulfonic acid (AIAS; Invitrogen) were added to the cvHsp solution to final concentrations of 8.5 and $3.2 \mathrm{mM}$, respectively, and incubated for $12 \mathrm{~h}$ at room temperature in the dark to fluorescently label the cysteine residues of cvHsp. The non-reactive probes were removed by passing the sample through a PD SpinTrap G-25 desalting column. Labeled cvHsp was eluted in the void volume of the column and pooled into a $1.5-\mathrm{ml}$ tube. The incorporation of AIAS and LYI into cvHsp was calculated from their absorption spectra using molar extinction coefficients of $35,000 \mathrm{~cm}^{-1} \mathrm{M}^{-1}$ at $335 \mathrm{~nm}$ and $13,000 \mathrm{~cm}^{-1} \mathrm{M}^{-1}$ at $435 \mathrm{~nm}$, respectively. The excitation maxima of AIAS-labeled and LYI-labeled cvHsps were 335 and $435 \mathrm{~nm}$, respectively, and their emission maxima were 415 and $525 \mathrm{~nm}$, respectively. To initiate the subunit exchange reaction, AIAS- and LYI-labeled cvHsps at the concentration of $10 \mu \mathrm{M}$ were mixed together at identical molarities in a tube 
A

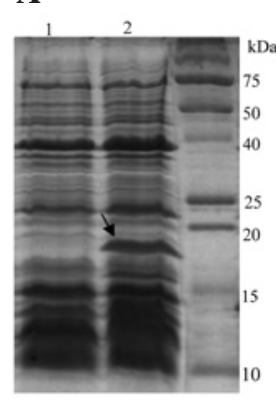

B

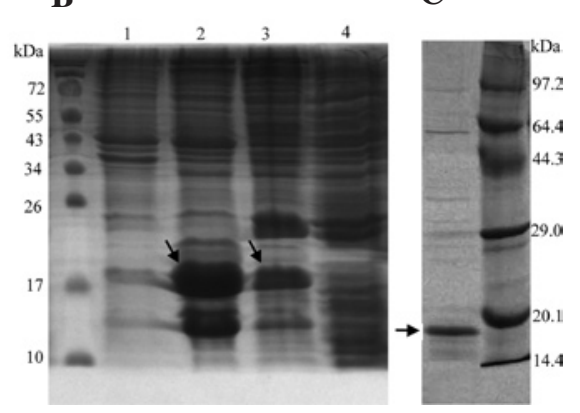

Figure 1. cvHsp overexpressed in E. coli and fractionated by saturated ammonium sulfate precipitation. (A) Inducible expression of cvHsp. Lane 1, total soluble protein from $E$. coli harboring the pET21a-crHsp plasmid; 2, total soluble protein from E. coli harboring the pET21a-cvHsp plasmid after a 4-h induction by $1 \mathrm{mM}$ IPTG. The resultant band was predicted to be $18 \mathrm{kDa}$. (B) SDS-PAGE of the fractions from saturated ammonium sulfate precipitation. Lanes 1, 2, 3 and 4 are the fractions precipitated by 20,40,60, and $80 \%$ saturated ammonium sulfate, respectively. (C) The pooled fraction of purified cvHsp following gel filtration chromatography.

at $60^{\circ} \mathrm{C}$. Aliquots $(10 \mu \mathrm{l})$ were removed from the reaction mixtures at various time points $(2,10,30$, and $50 \mathrm{~min})$ and diluted to $2 \mathrm{ml}$ in a quartz cuvette with $20 \mathrm{mM}$ PBS (pH 7.4) containing $0.15 \mathrm{M} \mathrm{NaCl}$. The fluorescence spectra were recorded at $37^{\circ} \mathrm{C}$ in the range of 400 to $600 \mathrm{~nm}$ with an excitation wavelength of $335 \mathrm{~nm}$. The excitation and emission band slits were both set at $2 \mathrm{~nm}$. The ratios of emission intensity of AIAS-labeled cvHsp at $415 \mathrm{~nm}$ to those of LYI-labeled cvHsp were plotted against the various time intervals. The normalized donor fluorescence intensity as a function of time, $F(t)$, was fit to the equation $\mathrm{F}(\mathrm{t})=\mathrm{C}_{1}+\mathrm{C}_{2} \mathrm{e}^{\mathrm{ekt}}$. The constants $\mathrm{C}_{1}$ and $\mathrm{C}_{2}$ were determined by the condition at which $\mathrm{C}_{1}+\mathrm{C}_{2}=1$ at $\mathrm{F}(0)$ and $\mathrm{F}$ (infinity) $=\mathrm{C}_{1}$. The rate constant $k$ was determined by nonlinear regression analysis of the data using the Microcal Origin 7.5 software.

\section{Results}

Gene cloning and protein purification. To incorporate restriction sites into the ends of the cvHsp gene, the resultant 550-bp PCR product was identified by agarose electrophoresis. The final PCR product was purified and ligated directly into the pMD18-T simple vector. The sequence of the cvHsp gene was confirmed by DNA sequencing (Genbank accession no. nm_031607). The resultant cvHsp fragment was ligated directly into the pET21a vector, which was previously digested with $N d e \mathrm{I}$ and EcoRI restriction enzymes to construct the pET21a-cvHsp plasmid, which was transformed into competent $E$. coli Rosetta (DE3) pLysS cells. The crude extract prepared from IPTG-induced bacteria exhibited obviously stained bands that corresponded in size to $18 \mathrm{kDa}$ in the SDS-PAGE gel (Fig. 1A). E. coli Rosetta (DE3) pLysS strain is an efficient prokaryotic expression system and yields a high expression level of the cvHsp gene. The supernatant of the crude extract was effectively fractionated by the different fractions of saturated ammonium sulfate. The bulk of cvHsp was found in the sediment of 40 and $60 \%$ saturated ammonium sulfate fractions (Fig. 1B). The fractions eluted at $0.15 \mathrm{M} \mathrm{NaCl}$ from Q-Sepharose anion exchange column were

A

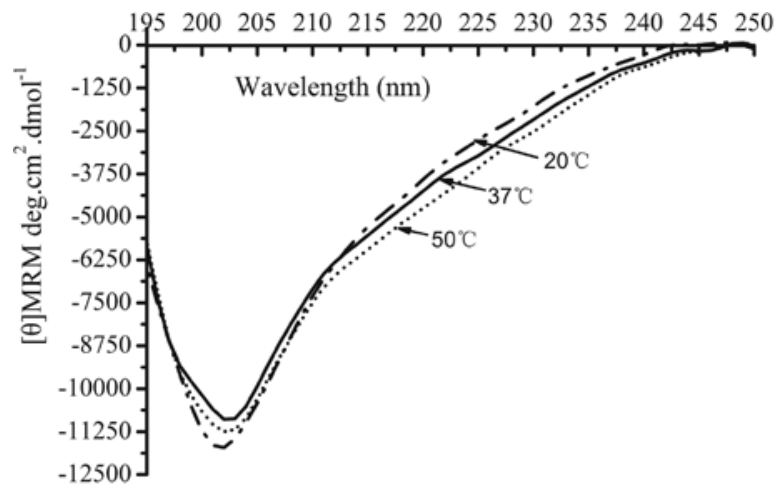

B

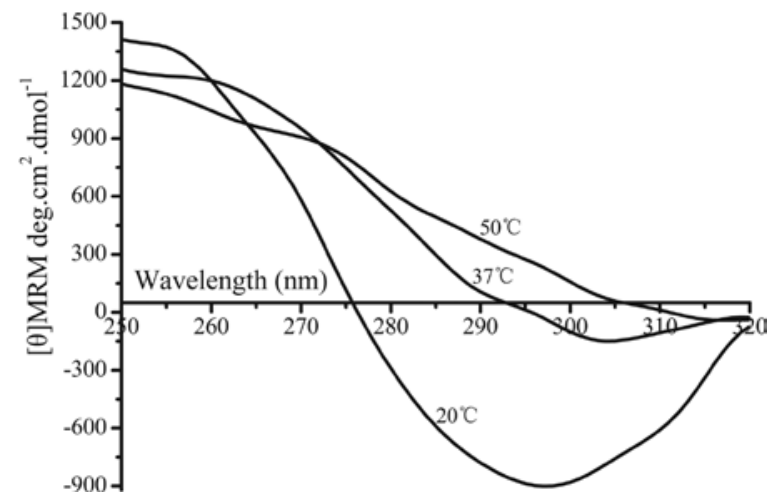

Figure 2. Far-UV and near-UV CD spectra of cvHsp. (A) Far UV-CD spectra at 20,37 and $50^{\circ} \mathrm{C}$. (B) Near UV-CD spectra at 20,37 and $50^{\circ} \mathrm{C}$. All spectra are recorded at a concentration of $0.20 \mathrm{mg} / \mathrm{ml}$ in $50 \mathrm{mM}$ PBS (pH 7.4). [ $\theta]$ MRM indicates the mean residue molar ellipticity.

found to contain recombinant cvHsp and were finally purified to homogeneity in a Superdex75 10/300GL column. After two chromatography procedures, the purity of recombinant cvHsp was estimated to be higher than $90 \%$ by SDS-PAGE analysis (Fig. 1C).

Secondary and tertiary structures. Circular dichroism was utilized to study the thermal transition of the molecular structure of cvHsp in the low range of protein concentrations, which may reflect relative alterations of the high order structure, which may in turn affect the related functionality of cvHsp. Fig. 2 displays a series of wavelength scans of $\mathrm{CD}$ spectra at different temperatures. The far-UV CD spectrum of cvHsp showed a marked negative ellipticity with a peak at $204 \mathrm{~nm}$, suggesting that $\mathrm{cvHsp}$ predominantly possesses $\beta$-sheet and randomly coiled structures. There was little difference in the spectral features at different temperatures $(20,37$ and $50^{\circ} \mathrm{C}$ ) (Fig. 2A). The essential secondary structural elements remained relatively unaltered at these temperatures. Using the CDPro deconvolution software, the secondary structural content was estimated to be 33\% $\beta$-sheet, $18 \% \beta$-turn, $30.5 \%$ random coil, and $18.5 \% \alpha$-helix. This was in agreement with the structural characteristics of other sHsp members that contain mainly $\beta$-sheet elements in the $\alpha$-crystallin domain and unstructured and $\alpha$-helical regions in the $\mathrm{N}$-terminal region (17).

Generally, the vibronic transition beyond $285 \mathrm{~nm}$ arises from Trp residues, whereas the bands between 285 and $275 \mathrm{~nm}$ reflect the molecular packing of Trp and Tyr residue side chains (18). The cvHsp does not contain Trp residue 
A

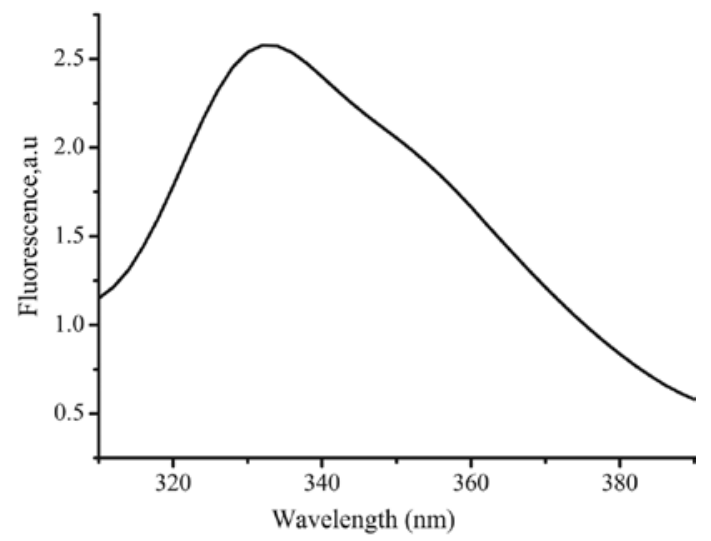

B

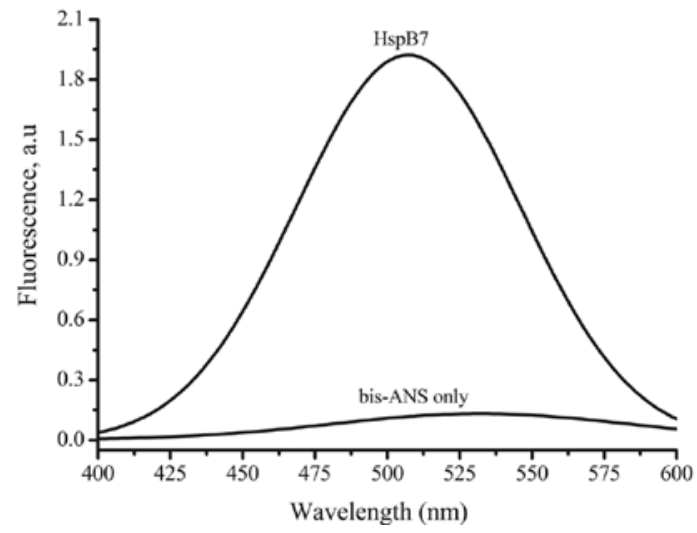

Figure 3. Fluorescence spectra of cvHsp. (A) Intrinsic fluorescence spectrum of cvHsp at a concentration of $0.225 \mathrm{mg} / \mathrm{ml}$. (B) bis-ANS-bound fluorescence spectrum of cvHsp at a concentration of $0.0225 \mathrm{mg} / \mathrm{ml}$.

in its sequence, which suggests that the subtle molecular packing-induced alterations were contributed by Tyr residue side chains. The near-UV CD spectra in Fig. 2B indicate that the side chain packing of the aromatic residues of $\mathrm{cvHsp}$ are significantly different at 20,37 and $50^{\circ} \mathrm{C}$. At $20^{\circ} \mathrm{C}$, the near-UV CD spectra of cvHsp exhibited a significantly positive molecular ellipticity compared to the spectra at 37 and $50^{\circ} \mathrm{C}$, the ellipticity of which was negative in the range between 285 and $275 \mathrm{~nm}$. This suggests that the Tyr residue side chain of cvHsp is buried in the tertiary structure, and that the molecular packing of the tertiary structure is variable and concomitant with temperature alterations. These temperatureinduced alterations represent the microenvironment of the Tyr residue side chain, and reflect a substantial alteration in the tertiary structure of cvHsp.

Fluorescence properties of cvHsp. In general, the maximum fluorescence intensity of Tyr residues was observed at $334 \mathrm{~nm}$. The cvHsp showed evident fluorescence intensity with an emission maximum at $332 \mathrm{~nm}$. The blue shift of $2 \mathrm{~nm}$ was possibly derived from the Tyr residue side chain in the $\mathrm{N}$-terminal region buried in the the molecular packing of tertiary structure (Fig. 3A). This result was presumably consistent with the intrinsic fluorescence of the conserved $\alpha$-crystallin domain in sHsp family members (19-21). The intrinsic fluorescence not only reflects the tertiary structure, but is also associated with the quaternary structure. The deduced amino acid sequence of the cvHsp gene contains
10 Phe residues, most of which are situated at the N-terminus, and one Tyr residue, situated at position 85. As a rule, some hydrophobic residues in the $\mathrm{N}$-terminal region of $\mathrm{sHsp}$ are capable of enhancing chaperone-like activity and bind various client proteins by hydrophobic interactions (22). Given this fact, one could hypothesize that cvHsp possesses the same chaperone-like activity in nature as other members of the sHsp family.

To determine whether the hydrophobic patches are localized on the molecular surface of cvHsp, the fluorescent probe bis-ANS was used to interact with the solvent-exposed surface of cvHsp and to probe the molecular hydrophobicity in solution. Upon binding to apolar surfaces, the fluorescence intensity of bis-ANS was enhanced with a blue shift of the emission maximum (23). As seen in Fig. 3B, the fluorescence intensity of bis-ANS after binding to cvHsp was significantly higher than bis-ANS alone, and exhibited a blue shift of emission maximum from $550 \mathrm{~nm}$ of bis-ANS to $500 \mathrm{~nm}$ of bis-ANS-bound cvHsp. This indicates that bis-ANS binds to the hydrophobic patches on the molecular surface of cvHsp. The cvHsp thus has some solvent-exposed hydrophobicity on the molecular surface.

Molecular architecture and subunit exchange of cvHsp. Mammalian sHsp often form several types of oligomers with unique molecular architecture, and exhibit dynamic molecular assembly. The multiple oligomeric state is in an equilibrium that can be manipulated by temperature, protein concentration and other inherent properties, such as phosphorylation and residue charge (24). A total of $90 \mu \mathrm{g}$ of cvHsp was applied to a Superdex $7510 / 300$ GL column, which produced two distinct peaks that were evaluated by molecular mass markers (Fig. 4). The first peak had a molecular mass of $\sim 220 \mathrm{kDa}$, which was an oligomer predicted to consist of 12 monomers. The second peak had a mass of $\sim 40 \mathrm{kDa}$, which was assumed to be a dimer with 2 monomers. According to the peak area calculation of Fig. 4A, one third of the total cvHsp content presumably exists as dimers, while the remaining components are oligomers. The pooled peak fractions were both assayed by SDS-PAGE under reducing conditions, and the same bands at $18 \mathrm{kDa}$ were observed (Fig. 4B). This confirms that cvHsp exists in an equilibrium state of two oligomeric forms. Therefore, cvHsp exhibits polydispersed molecular architecture in vitro.

FRET is a conventional strategy to determine whether sHsps have a dynamic molecular assembly characterized by a rapid subunit exchange between oligomers at physiological or higher temperatures. The subunit exchange reactions of $\alpha \mathrm{A}$-crystallin and $\alpha \mathrm{B}$-crystallin were previously monitored using AIAS and LYI as probes (25-27). The cvHsp protein contains a conserved $\alpha$-crystallin domain and exists as a mixture of the dimer and the large oligomer. Therefore, one population of cvHsp can be labeled with AIAS, which serves as the fluorescent donor, and another population of cvHsp can be labeled with LYI, which acts as the fluorescent acceptor. The covalent reaction mixture was applied to a Superdex G-25 desalting column to eliminate unbound probes. The cysteine residue of cvHsp was efficiently labeled by AIAS or LYI. When AIAS-labeled cvHsp was mixed with LYI-labeled cvHsp at an equimolar concentration in a 
A

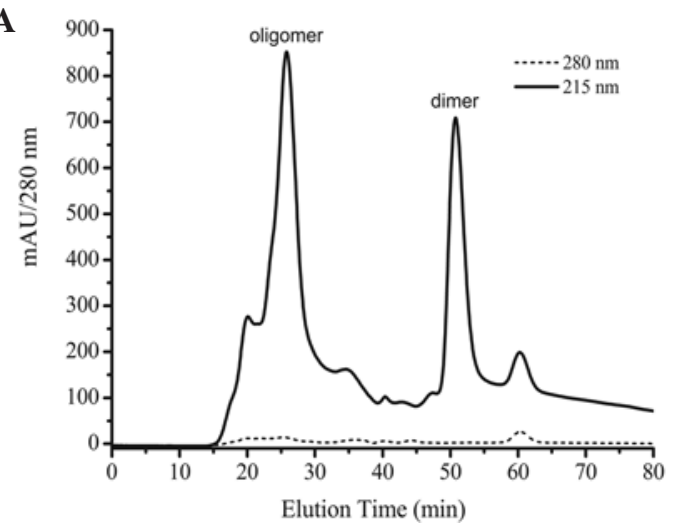

B

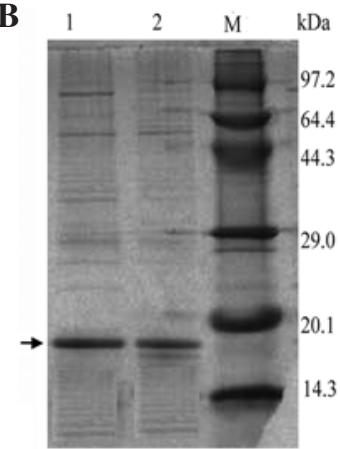

C

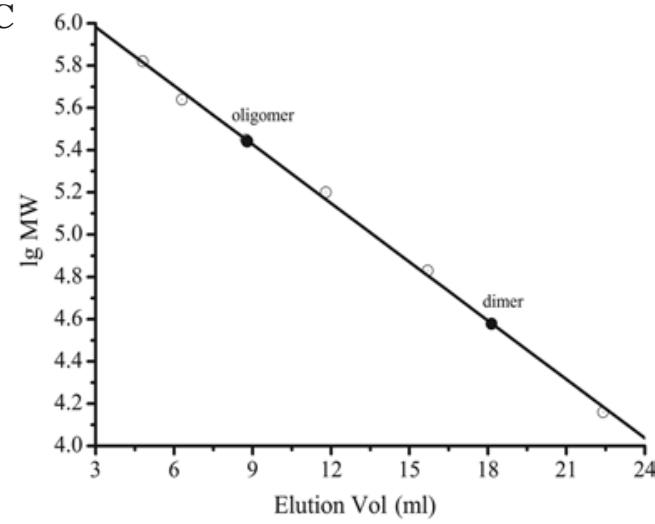

Figure 4. The molecular architecture of cvHsp. (A) The cvHsp $(90 \mu \mathrm{g}$ ) was applied to a Superdex75 10/300GL (Tricorn) column. Flow rate: $0.30 \mathrm{ml} / \mathrm{min}$, detection wavelengths: 280 and $215 \mathrm{~nm}$. (B) SDS-PAGE analysis of the peak fractions of oligomers and dimers; lane 1 and 2 show that the peaks derived from oligomers and dimers have the same-sized bands; $\mathrm{M}$ denotes molecular mass markers. The arrow denotes cvHsp. (C) The cvHsp and the standard curve of molecular mass markers in the Superdex75 10/300GL column.

A

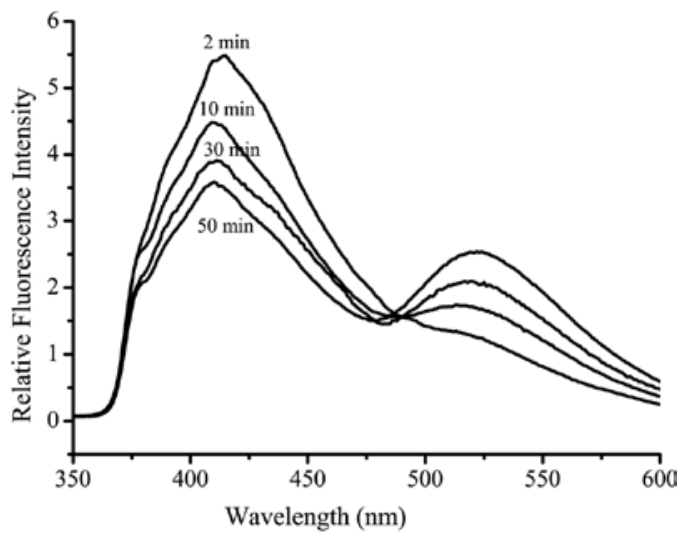

B

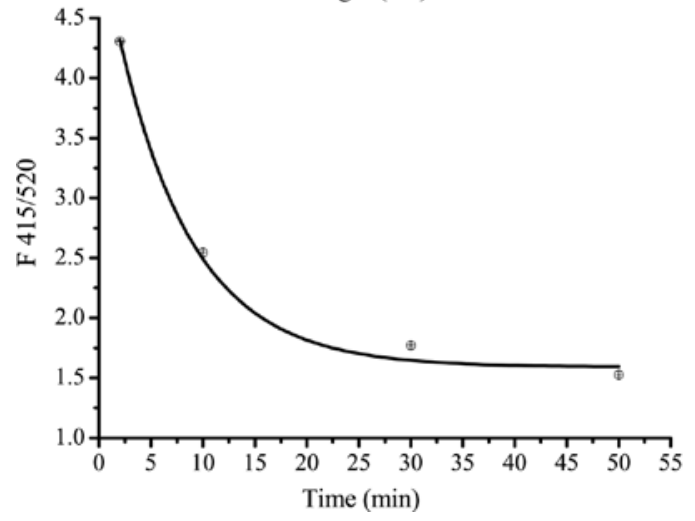

Figure 5. The subunit exchange process of cvHsp at $37^{\circ} \mathrm{C}$. (A) Timedependent FRET due to subunit exchange of AIAS-labeled and LYI-labeled cvHsp. The decrease in the fluorescence intensity of AIAS-labeled cvHsp at $415 \mathrm{~nm}$, and the concomitant increase in the fluorescence intensity of LYIlabeled cvHsp at $520 \mathrm{~nm}$, are indicative of energy transfer due to subunit exchange between the labeled populations. (B) The ratios of donor fluorescence intensity at $415 \mathrm{~nm}$ to the acceptor fluorescence intensity at $520 \mathrm{~nm}$ are plotted as a function of time. The exponential decay curve represents the statistical fit of the data to the function $F(t)=C_{1}+C_{2} e^{-k t}$.

cuvette at $60^{\circ} \mathrm{C}$, the subunit exchange reaction was initiated. A significant amount of quenching of the donor fluorescence was accompanied by a concomitant increase in the acceptor fluorescence, which was observed at the defined time points
(Fig. 5A). The subunit exchange reaction between oligomers brought the donor and the acceptor fluorophores close to each other, which allowed AIAS-labeled cvHsp to integrate into LYI-labeled cvHsp oligomers as the reaction progressed. The quenching of the donor fluorescence continued until $60 \mathrm{~min}$, after which further changes in the emission spectra were not observed. The ratio of the donor fluorescence intensity at $415 \mathrm{~nm}$ to the acceptor fluorescence intensity at $520 \mathrm{~nm}$ was plotted against the time points (Fig. 5B). Using a statistical program, quenching of the donor fluorescence was fit to the single exponential function $\mathrm{F}(\mathrm{t})=\mathrm{C}_{1}+\mathrm{C}_{2} \mathrm{e}^{-\mathrm{kt}}$, where the rate constant $k$ for subunit exchange was determined to be $0.042 \mathrm{~min}^{-1}$. This value was in agreement with that of the other known sHsps (28).

\section{Discussion}

Among sHsps expressed in cardiac tissue, the cvHsp may play a key role in cardiac metabolism (29). Previous reports have revealed that cvHsp is the most abundant transcript in heart libraries and may have extensive physiological implications $(9,30)$. Recent investigations have implicated cvHsp in oligomerization with other sHsps and cytoskeletal proteins in the myocardium. The cvHsp was previously found to form homo-oligomers as well as hetero-oligomers with HspB8 in myogenic tissues (31). The present study revealed that cvHsp, expressed in the E. coli Rosetta (DE3) pLysS strain, is a oligomeric protein with secondary, tertiary and quaternary structure, which exists in a mixture of oligomer and dimer in solution. It is presumably possible that the dimer represented a dissociated form of the large oligomer. The most notable feature of cvHsp is its capacity to exchange subunits between oligomers in vitro. The tertiary structure of cvHsp exhibited thermodynamics characteristic of molecular Tyr residue side chains, which contributed to the molecular functionality and molecular basis of the high order structure. The single cysteine of cvHsp was situated at position 125 in the C-terminal domain, which was similar to Hsp27, suggesting that this position is responsible for the close contact between two monomers, which also supports the notion that the dimer 
serves as the building block or minimum cooperative unit for the big oligomer of cvHsp. Human Hsp27 was found to form a large oligomer with an average mass of $530 \mathrm{kDa}$, which corresponds with an oligomer of approximately 24 subunits and confers cytoprotection against stress. Hsp27 also assembles into a small oligomer that is responsible for the stabilization of actin filaments and a large oligomeric architecture that exerts chaperone activity and a protective function (32). Recombinant Hsp27 was found to self-associate and form a highly dynamic oligomeric architecture that generates species as large as 12 and 16 monomers as well as smaller oligomers, which are probably tetramer, monomer and dimer. The chaperone-like activity and other physiological roles of Hsp27 were also found to depend on the self-associated state (33). In the present study, the cvHsp exhibited a similar molecular architecture to Hsp27 at high order structural hierarchy.

The fact that six or more rather similar sHsps have evolved to be expressed in the same tissues, notably heart and striated muscle, is intriguing. The reported paradigm to date is that each protein has its own important function $(34,35)$. Our present data identified various biochemical properties of cvHsp; however, whether and how these features affect functionality in vivo remains to be demonstrated. Duchenne muscular dystrophy is the most commonly inherited neuromuscular disorder in humans, but how pathophysiological events trigger fiber degeneration is still unclear. A proteomics analysis of normal diaphragm muscle versus that of patients with severely affected X-linked muscular dystrophy (MDX) indicated that the diaphragms of MDX patients had a drastic increase in the expression of the cvHsp gene, which was assumed to be an important functional protein for dystrophin-deficient fiber. Although other proteins also showed differential expression patterns, the most significant finding was the dramatic increase in the level of cvHsp in MDX fibers, which appears to be an important cellular mechanism in dystrophic muscle and may be utilized to counteract muscle degeneration (36-38). The cardiac cvHsp gene was found to play an important role in the cardiac stress response. Investigation of the phosphorylation states of cvHsp is currently in progress, and may aid in the clarification of the biological implications of the cvHsp gene.

\section{Acknowledgements}

This investigation was supported by the National 985 Key Project of Sichuan University of the Education ministry of China. We would like to thank Mrs. Zhihua Xing for her technical assistance with the CD spectrum instrument.

\section{References}

1. Vos MJ, Hageman J, Carra S and Kampinga HH: Structural and functional diversities between members of the human HSPB HSPH, HSPA, and DNAJ chaperone families. Biochemistry 47: 7001-7011, 2008

2. Treweek TM, Ecroyd H, Williams DM, Meehan S, Carver JA and Walker MJ: Site-directed mutations in the C-terminal extension of human alphaB-crystallin affect chaperone function and block amyloid fibril formation. PLoS One 2: e1046, 2007.

3. Shashidharamurthy R, Koteiche HA, Dong J and McHaourab HS Mechanism of chaperone function in small heat shock proteins: dissociation of the HSP27 oligomer is required for recognition and binding of destabilized T4 lysozyme. J Biol Chem 280: 5281-5289, 2005
4. Bova MP, Huang Q, Ding L and Horwitz J: Subunit exchange, conformational stability, and chaperone-like function of the small heat shock protein 16.5 from Methanococcus jannaschii. J Biol Chem 277: 38468-38475, 2002.

5. McHaourab HS, Dodson EK and Koteiche HA: Mechanism of chaperone function in small heat shock proteins. Two-mode binding of the excited states of T4 lysozyme mutants by alphaAcrystallin. J Biol Chem 277: 40557-40566, 2002.

6. Koteiche HA and McHaourab HS: Mechanism of chaperone function in small heat-shock proteins. Phosphorylation-induced activation of two-mode binding in alphaB-crystallin. J Biol Chem 278: 10361-10367, 2003.

7. Kappe G, Franck E, Verschuure P, Boelens WC, Leunissen JA and de Jong WW: The human genome encodes 10 alpha-crystallin-related small heat shock proteins: HspB1-10. Cell Stress Chaperones 8: 53-61, 2003.

8. De Wit NJ, Verschuure P, Kappe G, et al: Testis-specific human small heat shock protein HSPB9 is a cancer/testis antigen, and potentially interacts with the dynein subunit TCTEL1. Eur J Cell Biol 83: 337-345, 2004.

9. Krief S, Faivre JF, Robert P, et al: Identification and characterization of cvHsp. A novel human small stress protein selectively expressed in cardiovascular and insulin-sensitive tissues. J Biol Chem 274: 36592-36600, 1999

10. Vos MJ, Kanon B and Kampinga HH: HSPB7 is a SC35 speckle resident small heat shock protein. Biochim Biophys Acta 1793: 1343-1353, 2009

11. Matkovich SJ, Van Booven DJ, Hindes A, et al: Cardiac signaling genes exhibit unexpected sequence diversity in sporadic cardiomyopathy, revealing HSPB7 polymorphisms associated with disease. J Clin Invest 120: 280-289, 2010.

12. Pace CN, Vajdos F, Fee L, Grimsley G and Gray T: How to measure and predict the molar absorption coefficient of a protein. Protein Sci 4: 2411-2423, 1995.

13. Sreerama N and Woody RW: Estimation of protein secondary structure from circular dichroism spectra: comparison of CONTIN, SELCON, and CDSSTR methods with an expanded reference set. Anal Biochem 287: 252-260, 2000.

14. Bova MP, Ding LL, Horwitz J and Fung BK: Subunit exchange of alphaA-crystallin. J Biol Chem 272: 29511-29517, 1997.

15. Bova MP, McHaourab HS, Han Y and Fung BK: Subunit exchange of small heat shock proteins. Analysis of oligomer formation of alphaA-crystallin and $\mathrm{Hsp} 27$ by fluorescence resonance energy transfer and site-directed truncations. J Biol Chem 275: 1035-1042, 2000.

16. Ahmad MF, Raman B, Ramakrishna T and Rao Ch M: Effect of phosphorylation on alpha B-crystallin: differences in stability, subunit exchange and chaperone activity of homo and mixed oligomers of alpha B-crystallin and its phosphorylation-mimicking mutant. J Mol Biol 375: 1040-1051, 2008.

17. McHaourab HS, Godar JA and Stewart PL: Structure and mechanism of protein stability sensors: chaperone activity of small heat shock proteins. Biochemistry 48: 3828-3837, 2009.

18. Kundu M, Sen PC and Das KP: Structure, stability, and chaperone function of alphaA-crystallin: role of N-terminal region. Biopolymers 86: 177-192, 2007.

19. Sun Y, Bojikova-Fournier S and MacRae TH: Structural and functional roles for beta-strand 7 in the alpha-crystallin domain of p26, a polydisperse small heat shock protein from Artemia franciscana. FEBS J 273: 1020-1034, 2006.

20. Santhoshkumar P, Murugesan R and Sharma KK: Deletion of (54)FLRAPSWF(61) residues decreases the oligomeric size and enhances the chaperone function of alphaB-crystallin. Biochemistry 48: 5066-5073, 2009.

21. Kasakov AS, Bukach OV, Seit-Nebi AS, Marston SB and Gusev NB: Effect of mutations in the beta5-beta7 loop on the structure and properties of human small heat shock protein HSP22 (HspB8, H11). FEBS J 274: 5628-5642, 2007.

22. Seale JW, Gorovits BM, Ybarra J and Horowitz PM: Reversible oligomerization and denaturation of the chaperonin GroES. Biochemistry 35: 4079-4083, 1996.

23. Musci G, Metz GD, Tsunematsu H and Berliner LJ: 4,4'-Bis[8(phenylamino)naphthalene-1-sulfonate] binding to human thrombins: a sensitive exo site fluorescent affinity probe. Biochemistry 24: 2034-2039, 1985.

24. Fonte V, Kipp DR, Yerg J III, et al: Suppression of in vivo betaamyloid peptide toxicity by overexpression of the HSP-16.2 small chaperone protein. J Biol Chem 283: 784-791, 2008.

25. Kallur LS, Aziz A and Abraham EC: C-terminal truncation affects subunit exchange of human alphaA-crystallin with alphaB-crystallin. Mol Cell Biochem 308: 85-91, 2008. 
26. Ghahghaei A, Rekas A, Price WE and Carver JA: The effect of dextran on subunit exchange of the molecular chaperone alphaAcrystallin. Biochim Biophys Acta 1774: 102-111, 2007.

27. Liang JJ and Liu BF: Fluorescence resonance energy transfer study of subunit exchange in human lens crystallins and congenital cataract crystallin mutants. Protein Sci 15: 1619-1627, 2006.

28. Gupta R and Srivastava OP: Deamidation affects structural and functional properties of human alphaA-crystallin and its oligomerization with alphaB-crystallin. J Biol Chem 279: 44258-44269, 2004.

29. Taylor RP and Benjamin IJ: Small heat shock proteins: a new classification scheme in mammals. J Mol Cell Cardiol 38: 433-444, 2005.

30. Doran P, Gannon J, O'Connell K and Ohlendieck K: Aging skeletal muscle shows a drastic increase in the small heat shock proteins alphaB-crystallin/HspB5 and cvHsp/HspB7. Eur J Cell Biol 86: 629-640, 2007.

31. Sun X, Fontaine JM, Rest JS, Shelden EA, Welsh MJ and Benndorf R: Interaction of human HSP22 (HSPB8) with other small heat shock proteins. J Biol Chem 279: 2394-2402, 2004.

32. Rogalla T, Ehrnsperger M, Preville X, et al: Regulation of Hsp27 oligomerization, chaperone function, and protective activity against oxidative stress/tumor necrosis factor alpha by phosphorylation. J Biol Chem 274: 18947-18956, 1999.
33. Lelj-Garolla B and Mauk AG: Self-association and chaperone activity of Hsp27 are thermally activated. J Biol Chem 281: 8169-8174, 2006

34. Horwitz J, Huang Q and Ding L: The native oligomeric organization of alpha-crystallin, is it necessary for its chaperone function? Exp Eye Res 79: 817-821, 2004.

35. Van de Klundert FA, Smulders RH, Gijsen ML, et al: The mammalian small heat-shock protein $\mathrm{Hsp} 20$ forms dimers and is a poor chaperone. Eur J Biochem 258: 1014-1021, 1998.

36. Doran P, Wilton SD, Fletcher S and Ohlendieck K: Proteomic profiling of antisense-induced exon skipping reveals reversal of pathobiochemical abnormalities in dystrophic MDX diaphragm. Proteomics 9: 671-685, 2009.

37. Doran P, Martin G, Dowling P, Jockusch H and Ohlendieck K: Proteome analysis of the dystrophin-deficient MDX diaphragm reveals a drastic increase in the heat shock protein cvHSP. Proteomics 6: 4610-4621, 2006.

38. Golenhofen N, Perng MD, Quinlan RA and Drenckhahn D: Comparison of the small heat shock proteins alphaB-crystallin, MKBP, HSP25, HSP20, and cvHSP in heart and skeletal muscle. Histochem Cell Biol 122: 415-425, 2004. 